\title{
Anabases
}

ANABASES Traditions et réceptions de l'Antiquité

Stéphane RATTI, Antiquus error. Les ultimes feux de la résistance païenne Scripta varia augmentés de cinq études inédites

\section{Christian Stein}

\section{(2) OpenEdition}

1 Journals

Édition électronique

URL : http://journals.openedition.org/anabases/2419

DOI : 10.4000/anabases.2419

ISSN : 2256-9421

Éditeur

E.R.A.S.M.E.

\section{Édition imprimée}

Date de publication : 1 octobre 2011

Pagination : 283-284

ISSN : 1774-4296

\section{Référence électronique}

Christian Stein, «Stéphane ratTI, Antiquus error. Les ultimes feux de la résistance païenne Scripta varia augmentés de cinq études inédites », Anabases [En ligne], 14 | 2011, mis en ligne le 01 octobre 2011, consulté le 21 septembre 2020. URL : http://journals.openedition.org/anabases/2419 ; DOI : https:// doi.org/10.4000/anabases. 2419

Ce document a été généré automatiquement le 21 septembre 2020

(c) Anabases 


\title{
Stéphane RATTI, Antiquus error. Les ultimes feux de la résistance païenne Scripta varia augmentés de cinq études inédites
}

\author{
Christian Stein
}

\section{RÉFÉRENCE}

Stéphane RATTI, Antiquus error. Les ultimes feux de la résistance païenne Scripta varia augmentés de cinq études inédites, Turnhout, Brepols Publishers, 2010, 328 p.

40 euros / ISBN : 978-2-503-53261-5.

1 Stéphane Ratti (S.R.), professeur de langue et littérature latines à l'université de Bourgogne (Dijon) et spécialiste de l'Antiquité tardive, offre avec Antiquuus error une collection de 35 travaux rédigés entre 1996 et 2007. Après une préface de Jean-Michel Carrié et un bref avant-propos de l'auteur, l'ensemble est structuré en cinq parties (I-V) - elles-mêmes subdivisées en chapitres - qui sont autant de pôles autour desquels s'organise l'activité scientifique de S.R.: "Le quatrième siècle: historiographie et christianisme " ( 9 textes, dont 1 inédit); «Saint Jérôme et ses sources profanes " (8 textes + 1 inédit) ; "Ammien Marcellin et la tradition héroïque » (3); "L'Histoire Auguste: un faussaire démasqué » $(12+3)$; "Claudien, Rutilius Namatianus et les chrétiens » (3). On y trouve surtout des articles, quelques comptes rendus critiques mais aussi le discours de son habilitation à diriger des recherches. Quelques coquilles subsistent (par ex. p. 98 wat pour what, p. 105 Valentinen pour Valentinien etc.), mais l'ensemble est très agréablement présenté. Je ne comprends néanmoins pas trop pourquoi certains textes sont précédés d'un résumé en français, certains aussi d'un abstract, mais d'autres en revanche de rien du tout. Plus gênant, il y a peu de renvois internes au sein de l'ouvrage entre les différents chapitres, et, à nouveau, pourquoi le 
faire dans certains cas (au hasard : p. 217, n. 1 ou p. 235, n. 17) et pas dans d'autres (par ex. p. 174, n. 5) ? Le tout est complété par d'importants et utiles index (noms, auteurs et œuvres antiques, rerum) qui facilitent grandement la consultation de l'ouvrage.

Antiquus error se présente de loin comme un recueil de scripta varia, mais l'ouvrage forme un tout bien supérieur à la somme de ses parties. Il ne s'agit en effet pas ici d'une simple réédition de travaux anciens, réunis pour la commodité des lecteurs de S.R., mais de la mise en évidence d'une thèse qui sert depuis une quinzaine d'années de fil directeur plus ou moins discret à ses recherches, en apparence plus dispersées. En effet, S.R. travaille moins sur ses auteurs antiques de prédilection que sur une question beaucoup plus générale et ambitieuse : «Comment païens et chrétiens ont-ils cohabité entre l'année 370, qui est la date de publication du Bréviaire d'Eutrope, et 395 qui voit disparaître le principal auteur des mesures en faveur des chrétiens» (p.11), avec un prolongement jusqu'à l'époque de Rutilius Namatianus. À travers les différents chapitres de l'ouvrage, on constatera que le style est souvent moins celui de l'exposé que celui du débat: S.R. aime engager la discussion avec ses collègues. Ses argumentations sont toujours très claires et très démonstratives: le lecteur a donc toute latitude pour décider s'il rejoint les conclusions de l'auteur ou non. Globalement, le propos est toujours ferme et jamais mièvre : S.R. dit ce qu'il pense et n'hésite pas à être sévère, même si le ton reste toujours poli et le fond académique.

Antiquus error présente deux intérêts essentiels. Le premier relève de la Quellenforschung et concerne l'ensemble des discussions sur la généalogie des œuvres historiographiques $d u \mathrm{IV}^{\mathrm{e}}$ et $\mathrm{du} \mathrm{v}^{\mathrm{e}}$ siècle. Il faut y lire les multiples articles sur Eutrope, Jérôme et quelques autres : non qu'il soit nécessaire de systématiquement se rallier aux idées de S.R., mais simplement pour saisir, au moins partiellement, les complexités généalogiques de l'historiographie tardo-antique, afin d'utiliser ces sources de manière moins naïves qu'elles ne le sont parfois. S.R. a ainsi raison de pointer (p. 239) ce paradoxe cocasse que l'Histoire Auguste déroute encore tellement - malgré les importants travaux accomplis depuis deux générations - qu'on ne la propose toujours pas à l'agrégation de lettres classiques, alors que, d'un autre côté, le ministère la met quand même au programme du secondaire...

Dans ce cadre, le morceau de choix est évidemment tout ce qui tourne autour de ladite Histoire Auguste. On rappellera en effet que S.R. a créé la surprise en 2005 en proposant d'identifier l'auteur du recueil avec Nicomaque Flavien senior : s'est ouverte alors une belle polémique dont l'avenir dira (peut-être) qui a raison. Je déplore à ce propos l'absence dans Antiquus error de la réponse de S.R. aux critiques de François Paschoud, réponse évoquée p. 274, n. 25 (cf. S.R. « 394 : fin de la rédaction de l'Histoire Auguste?», Antiquité Tardive 16, 2008, p. 335-348) : les arguments y sont à mon avis beaucoup plus forts que ceux exposés dans IV, 9.

Mon seul regret sur cette partie historiographique est que l'organisation non linéaire du livre rend la compréhension des choses un peu délicate pour le lecteur qui n'est pas un sectateur de la Quellenforschung. Les articles ne sont en effet pas rangés dans l'ordre chronologique de publication, or la pensée de l'auteur a clairement évolué entre 1996 et aujourd'hui et il est parfois difficile de s'y retrouver. Pour ne prendre qu'un exemple, on lit p. 152 un "Or l'on sait désormais que...» qui s'appuie (n. 42) sur un texte n'apparaissant en fait dans le livre qu'à la page 217. À la limite, je recommanderai même de commencer la lecture de l'ouvrage par là ! 
6 Le deuxième intérêt d'Antiquus error est de participer pleinement à un travail de réhabilitation générale de la littérature tardive païenne. Beaucoup ne voient toujours dans Eutrope, Macrobe, Rutilius Namatianus et d'autres qu'une littérature sinon oiseuse, du moins essentiellement récréative. Ces auteurs auraient écrit simplement pour passer le temps ou éventuellement favoriser leur petite carrière personnelle par quelque flatterie littéraire. C'est oublier qu'ils appartenaient à des élites dont la vocation consciente était de commander et de s'engager dans l'activité politique : c'est avec cela à l'esprit qu'il faut aborder leurs écrits. Je pense par exemple à l'interprétation de la civilitas, très présente chez Eutrope et largement sous-estimée avant S.R., qui lui a restitué toute sa dimension politique (p.17-23 et p.44; je proposerais à ce propos pour Eutrope, VII, 8 de traduire plutôt vixit civilissime par « il vécut en citoyen parfait ", qui conserve, mieux que " il se comporta exactement comme un citoyen ordinaire ", la dimension superlative d'un hapax renvoyant tout de même à Auguste, un citoyen tout sauf ordinaire).

7 Évidemment la grande question politique de l'arc chronologique choisi par S.R. était la question de la coexistence religieuse, dans un contexte de progression implacable du christianisme, et il réussit parfaitement à montrer que les élites païennes n'étaient pas aussi décadentes et passives qu'on le dit parfois. Même si nous savons aujourd'hui qu'il ne s'est agi que d'un combat d'arrière-garde, la guerre des idées a bien fait rage.

\section{AUTEURS}

\section{CHRISTIAN STEIN}

Université de Bourgogne

stein.christian@free.fr 